\title{
Compensation in Pulsed Alternators
}

\author{
S. B. Pratap and M. D. Driga \\ Center for Electromechanics / Electrical and Computer Engineering Department \\ The University of Texas at Austin
}

\begin{abstract}
This paper presents a generic discussion on compensation in pulsed alternators. It covers all aspects of compensation i.e., types of compensation, alternative methods for the physical realization of the various compensation schemes, the pros and cons of compensation or the rationale for compensation The intent of this paper is to form a guiding reference on the issues of compensation in pulsed machinery.
\end{abstract}

\section{INTRODUCTION}

Pulsed alternators are being used as power supplies for various high power applications. These applications range from electromagnetic launchers, electrothermal chemical launchers and lasers. These pulsed alternators are air-core presently, although the first few machines that were built during the 70's and early 80 's were iron core. The shift to air core designs was driven by the need for higher energy density and higher power density. Carbon fiber composites (CFCs) with their higher strength to density ratios made this revolution in pulsed rotating machines possible. The shift to air core designs is possible because the pulsed machines do not operate in a continuous mode. Even though the excitation ampere-turns are greater as compared to the iron core designs, the time that the field coil is on is short thus the losses in the field coil are manageable. This implies that for most of the applications the field coil is charged in less than $100 \mathrm{~ms}$, the main discharge pulse completed, and the field energy reclaimed for every shot. Charging the field coil in a short time implies higher power needs. Exciting the field coil separately would require another high power alternator. Therefore, the air core designs are self-excited ("boot-strapped") either by the armature winding used for the high current discharge or an independent winding dedicated for the excitation process.

The machine topology has undergone an evolution and so has the operating mode. Earlier air core machines were built with the armature (high current) winding on the rotor and the more massive but lower current field coil on the stator. These earlier machines were designed to give a single pulse from the stator. The pulse shape could be tailored to the application from a very peaky pulse for the lasers to a flat-topped pulse

Manuscript received May 1, 1998

S. B. Pratap (512) 471-4496, fax (512) 471-0781, s.pratap@mail.utexas.edu, M. D. Driga (512) 471-6943.

This work was supported by U.S. Army ARDEC under contract no. DAAA21-92-C-0105. for the electromagnetic launchers. The pulse shape was determined by the characteristics of the compensation. The present class of machines are built with the field coil spinning on the rotor and with the armature winding on the stator. This shift was made possible by further increasing the charging rate of the field coil. Also, the operating mode of the machine was changed from single-phase, single-pulse to multi-phase multipulse. In the new mode of operation the machine has three to four phases wherein each phase is discharged in sequence into the load while synthesizing the required pulse shape to the load over a number of pulses. This mode of operation decouples the rotational speed from the pulse width thus allowing the machine rotational speed to be selected more optimally for higher energy density.

\section{COMPENSATION}

The single-phase, single-pulse machines were very dependent on the type of compensation provided to generate the desired pulse shape for the load. The types of compensation were active, passive, and selective passive. The active compensation provided a peaky pulse and constituted a compensating winding connected in series with the armature winding. The passive compensation provided a more or less sinusoidal output and was merely a continuous conductive shield which compressed the flux for all rotor positions. The selective passive compensation provides a wide variety of pulses including the flat-topped pulse. The selective passive compensation is in the form of a compensating winding shorted on itself with its magnetic axis tilted at the appropriate angle with respect to the axis of the field coil to generate a wide variety of pulse shapes. References [1], [2], and [3] are good sources to learn more about these class of machines and their compensation techniques.

The focus of this paper is on the compensation needs and methods of the newer class of machines i.e., the multi-phase, multi-pulse machines. It must be noted that the pulse shape in these machines is controlled by controlling the phase angle at which each of the phases is triggered into the load. The main task that the compensation has then is to reduce the internal inductance of the phases so as to facilitate the commutation of the phases. There are a few other duties that the compensation does, one of which is the reduction of overvoltages and the second is the reduction of losses in the field coil. These will be discussed in what follows. Difficulties and the negative aspects of compensation will also be discussed. 


\section{Compensation Issues}

To get the lowest inductance in the armature windings of the machine, it is desirable to provide compensation in the form of a continuous conductive shield in close proximity to the armature winding. Another significant advantage of this form of compensation is that the transient fields produced during discharge are kept away from the field coil. This is a desirable feature as then the field coil can be designed and optimized for the exclusive purpose of providing the excitation field. Whenever a component serves a dual purpose it usually represents a compromise on both functions.

The field coil needs to be charged fast to manage the losses. Any highly conductive material enclosing the field coil (such as the continuous conductive compensating shield) would retard the growth of the field strength near the armature winding. Thus, besides slowing down the charging process and increasing losses in the field coil, it also induces unwarranted losses in the conducting material. The type of compensation, if any, must therefore have minimum interaction with the field coil charging process.

These are the two main issues related to compensation in the multi-phase, multi-pulse machines regarding compensation. In order to address these issues, several options on how compensation may be provided are discussed below.

\section{A Circuit Representation of Compensation}

To understand compensation in its various forms consider the circuit diagram of Fig. 1. The armature winding is shown as a voltage source along with a series inductance. This corresponds to the uncompensated phase inductance of the armature coil.

Also shown in the circuit schematic are two additional coils which are the compensating windings. These windings have their magnetic axes inclined at an angle of $90^{\circ}$ to each other

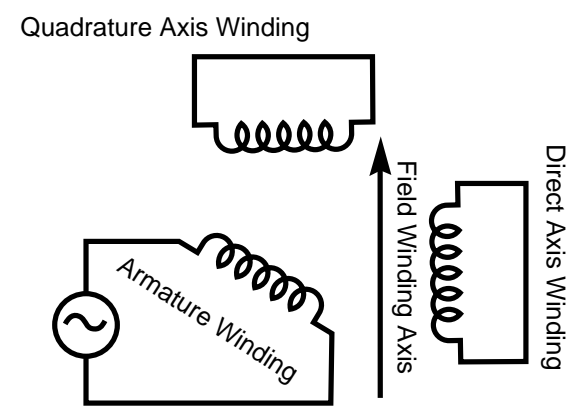

3910.0717

Fig. 1. Circuit model for a two axis compensated machine. so they do not couple. However, they are in close proximity to the armature winding and therefore couple well with it. As the armature spins relative to the compensating coils, the mutual inductance of the armature with these coils changes with rotor position:

$$
\begin{aligned}
& \mathrm{M}_{\mathrm{ac} 1}=\mathrm{M}_{\mathrm{mac} 1} \operatorname{Cos}(\theta) \\
& \mathrm{M}_{\mathrm{ac} 2}=\mathrm{M}_{\operatorname{mac} 2} \operatorname{Sin}(\theta)
\end{aligned}
$$

To determine the effective inductance of the armature as a function of rotor position, we write the circuit equations as follows:

$$
\left[\begin{array}{ccc}
\mathrm{L}_{\mathrm{a}} & \mathrm{M}_{\mathrm{ac} 1} & \mathrm{M}_{\mathrm{ac} 2} \\
\mathrm{M}_{\mathrm{ac} 1} & \mathrm{~L}_{\mathrm{c} 1} & 0 \\
\mathrm{M}_{\mathrm{ac} 2} & 0 & \mathrm{~L}_{\mathrm{c} 2}
\end{array}\right] \cdot\left[\begin{array}{c}
\frac{\mathrm{di}_{\mathrm{a}}}{\mathrm{dt}} \\
\frac{\mathrm{di}_{\mathrm{c} 1}}{\mathrm{dt}} \\
\frac{\mathrm{di}_{\mathrm{c} 2}}{\mathrm{dt}}
\end{array}\right]=\left[\begin{array}{l}
\mathrm{V} \\
0 \\
0
\end{array}\right]
$$

Solving for

$$
\frac{\mathrm{di}_{\mathrm{a}}}{\mathrm{dt}}
$$

we get:

$$
\frac{\mathrm{di}_{\mathrm{a}}}{\mathrm{dt}}=\frac{\mathrm{V}}{\mathrm{L}_{\mathrm{a}}\left\{1-\mathrm{k}^{2} \mathrm{acm} 2 \operatorname{Sin}^{2}(\theta)-\mathrm{k}^{2}{\left.\mathrm{acm} 1 \operatorname{Cos}^{2}(\theta)\right\}}^{2}\right.}
$$

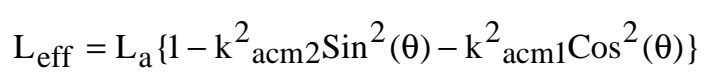

$\mathrm{L}_{\mathrm{eff}}$ is the effective phase inductance of the armature winding.

$$
\begin{aligned}
& \text { Here } \mathrm{k}_{\mathrm{acm} 1}=\frac{\mathrm{M}_{\mathrm{acm} 1}}{\sqrt{\mathrm{L}_{\mathrm{a}} \mathrm{L}_{\mathrm{c} 1}}} \text { and } \mathrm{k}_{\mathrm{acm} 2}=\frac{\mathrm{M}_{\mathrm{acm} 2}}{\sqrt{\mathrm{L}_{\mathrm{a}} \mathrm{L}_{\mathrm{c} 2}}} \\
& \text { i.e., the coupling coefficients }
\end{aligned}
$$

It is quite apparent from (1) that the effective inductance is lower compared to the uncompensated case. A special case to be considered is when $\mathrm{k}_{\mathrm{acm} 1}=\mathrm{k}_{\mathrm{acm} 2}$. The effective inductance then becomes $\mathrm{L}_{\text {eff }}=\mathrm{La}\left(1-\mathrm{k}_{2 \mathrm{acm}}\right)$. That is the effective armature inductance is lower by the factor $\left(1-\mathrm{k}_{2 \mathrm{acm}}\right)$ and is not a function of the relative rotor position. This indicates that it is possible to emulate a continuous conductive shield using two $90^{\circ}$ displaced windings for compensation. For the case where $\mathrm{k}_{\mathrm{acm} 1} \neq \mathrm{k}_{\mathrm{acm} 2}$, the effective armature inductance is not constant with rotor position. The extent of the variation in this case will depend upon the extent that the couplings differ as illustrated in (2) and also Fig. 2:

$$
\mathrm{L}_{\mathrm{eff}}=\mathrm{L}_{\mathrm{a}}\left\{1-\frac{\mathrm{k}^{2} \mathrm{acm} 1+\mathrm{k}^{2} \mathrm{acm} 2}{2}-\frac{\mathrm{k}^{2} \mathrm{acm} 1-\mathrm{k}^{2} \mathrm{acm} 2}{2} \operatorname{Cos}(2 \theta)\right\}(2)
$$




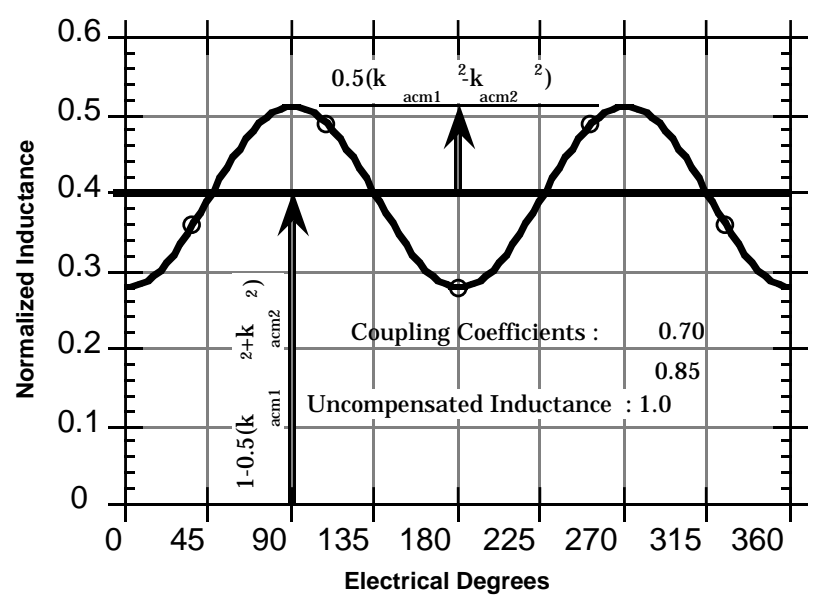

3910.0718

Fig. 2. Inductance variation with rotor position for twoaxis compensation.

The latter $\left(\mathrm{k}_{\mathrm{acm} 1} \neq \mathrm{k}_{\mathrm{acm} 2}\right)$ situation is applicable when the compensation, instead of being provided by a continuous conducting shield, is provided by a combination of the field coil itself and a compensating winding placed in quadrature (magnetic axes at $90^{\circ}$ electrical displacement) to the field coil. The field coil is typically thicker than the compensating winding because of the higher action requirements in the field coil. The coupling of the armature winding with the field coil is therefore lower than that with the compensating winding. Two-axis compensation can, however, be provided by this technique.

If the compensating winding is removed, then the field coil will compensate along only one axis (the direct-axis). The inductance variation then takes the shape shown in Fig. 3. It can be seen that the minimum inductance in this case is equal to the maximum inductance of the case with the compensating winding. Since there is no compensation along the axis at $90^{\circ}$ to the field coil, the effective inductance attains the full uncompensated value at this position and in general higher machine inductance is observed. In reality, if the compensating winding is removed, the field winding can be brought closer to the armature winding and a slight improvement in the field coil coupling is observed. The result is that the minimum effective armature inductance will be slightly lower when the field coil alone compensates.

\section{TYPES OF COMPENSATION}

\section{Two-Axis Wound/Switched Compensation}

As mentioned earlier, it is possible to emulate a continuous conductive shield with two compensating windings having

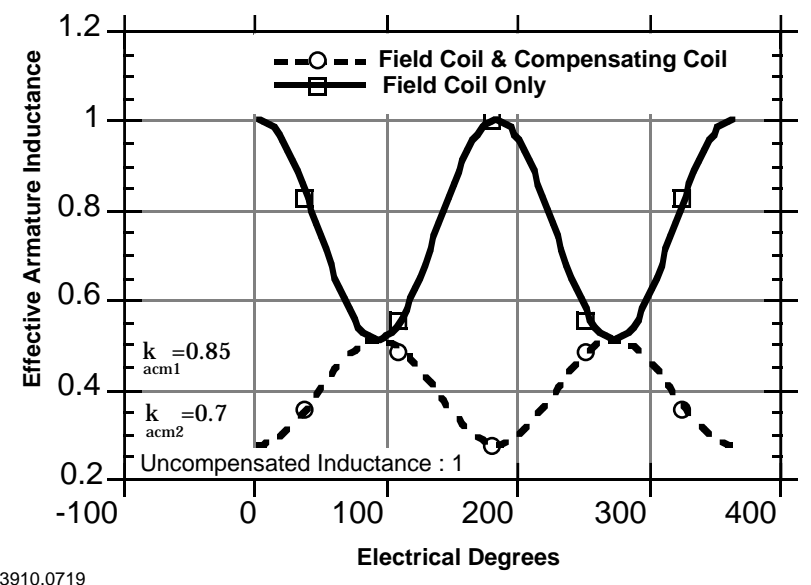

Fig. 3. Comparison of armature inductance variation with and without the compensating.

identical maximum coupling with the armature and with 90 electrical degrees phase displacement with each other. One of the compensating windings can be placed along the field coil axis (direct axis) and the other at 90 electrical degrees to it (quadrature axis). Now, during the field coil charging cycle, the quadrature axis compensating winding does not experience any transient induced current since the coupling is zero. The direct axis compensating winding if it were closed on itself, as is usually the case, would have induced currents in it due to its coupling with field coil. If, however, a switch is placed in the direct axis compensating winding circuit, then this switch can remain open during the field coil charging cycle and then closed just prior to the main gun discharge. This would eliminate the transient in the direct axis winding during charging of the field coil and provide the requisite compensation for the gun pulse. The machines being considered now are rotating field coil machines. This has several advantages one of which is that the field coil current is lower than the armature current thus simplifying the current collection system. The compensating winding is also on the rotor in these machines. Switching on and off one of the compensating windings would require an additional current collection system, which may not be very desirable. The number of turns in the compensating windings, and therefore the current that must be switched, can be determined independently. The turns can be adjusted so that the current collection system needs to handle low currents. However, the complication involved in coordinating an extra switching event is present in such a system.

The field coil in this case does not experience the transient high magnetic fields during the discharge as the windings along the two axes protect the field coil from this transient. 


\section{Quadrature Axis Compensating Winding}

This system of compensating the armature evolved as a solution to the switched two axis compensating technique. The main drawback of the previously discussed system was the switch in the direct axis compensating winding circuit. If the direct axis compensating winding is eliminated, there is no winding to couple with the field coil charging process. The task of compensating along the direct axis is now taken over by the field winding. The quadrature axis compensating winding is maintained as before. Since the field coil is designed for the action of the charging process as well as the discharge pulse into the gun its thickness will be greater than the compensating winding. The result is that the maximum coupling of the armature with the field winding is lower than the coupling with the quadrature axis compensating winding. The effective armature inductance variation is shown in Fig. 2. In general, the effective armature inductance is somewhat higher than that in the case of the switched two axis compensating windings. This scheme represents a judicious compromise between the need to fast charge the field coil and the need to provide effective compensation to the armature winding. However, the field coil does experience the high transient fields during the discharge.

\section{Thin Conductive Shell}

Typical charging times for the field coil range from $20 \mathrm{~ms}$ to $50 \mathrm{~ms}$ whereas the pulse width in the discharge (gun) circuit is in the range of $5 \mathrm{~ms}$ to $7 \mathrm{~ms}$. An option that arises considering these times is to design a continuous conductive shell thin enough so as to not significantly interact with the slower field coil charging process and yet provide effective compensation for the faster discharge pulse into the gun.

When this option is investigated in depth, it is found that to provide effective and efficient compensation the thickness of the compensating shield is such that it does interfere with the charging process. The reason for this condition can be explained in a simple way by considering the transient penetration depth, which varies as the square root of time. This tradeoff could be effectively made only in those cases where the charging time and discharge times are different by a few orders of magnitude.

Reference [4] gives an in depth analysis of the shield with and without motional effects.

\section{No Compensating Winding/Shell}

In this mode of compensation, there is no dedicated compensating winding. The field coil plays the dual role of providing excitation field and direct axis compensation. Therefore, in this case the question of interaction between the compensating winding and the field coil charging does not exist. However, significant overvoltages are observed in the armature windings. The overvoltages have implications on winding insulation and switch size and ratings. The absence of the compensating winding improves the coupling between the armature and the field coil which is beneficial during charging but also results in higher transient currents (and therefore higher losses) during discharge. The field coil experiences the high transient discharge fields and additionally the absence of the quadrature axis compensation means that the fields produced by the armature along the quadrature axis can penetrate all the layers of the field coil thus inducing greater losses.

\section{Compensating Winding as a Means for Suppressing Overvoltages}

To suppress overvoltages in high pulsed power alternators for railguns or coilguns, even an imperfect compensation structure is welcomed, especially when modern solid state devices are involved for two main reasons:

a) One is of general statistical nature: a very recent IEEE paper [5] shows that since the introduction of highly efficient fast-switching insulated gate bipolar devices (IGBTs) or other advanced solid state devices in controlling electrical machines, there has been documented a large increase of insulation breakdowns blamed on overvoltages, inherent to electronic commutation.

b) A second reason, of particular interest to compulsators supplying large current to electromagnetic accelerators during the launching; if such large currents flow only in one part of the armature windings, then in the rest of the armature a large overvoltage appears in the absence of a compensating winding. The overvoltages are suppressed by the solid rotor body and damper windings in iron core compulsators $[6,7]$ or by a rudimentary compensation winding in air core pulsed alternators.

The "uncompulsator" was proposed as a very high voltage pulsed electrical machine in which large voltages are induced in one part of the armature winding while the high short-circuit currents flow in the rest of the armature winding. It is the opposite (electromagnetic dual) of a compulsator in which not only the compensation winding is eliminated, but also all the other means of suppressing overvoltages are removed[7]. In iron core machines, this requires a finely laminated structure for the magnetic circuit, while in the air core machines, built of low conductivity composite materials, such a constraint is naturally enforced.

Its operation is based on a strong analogy with the "asymmetrical sudden short circuit" regime of synchronous generators. The regime is characterized as asymmetrical since the switch is closed at the moment of the maximum flux linkage in the machine and a dc component of the current appears to maintain constant such linkage, practically doubling the short circuit current after almost 180 electrical degrees. If, however, only two phases out of three are short-circuited (another asymmetry), the third phase will produce a very large voltage, many times the rated voltage of the normal synchronous generator.

The two short-circuited phases form a winding whose axis is perpendicular to the axis of the third (output) phase (Fig. 4). When the switch is closed, the two phases, B and C, are short- 
circuited and the event is similar to the sudden short-circuit of a conventional asynchronous machine. The fluxes, currents, and electromotive forces add vectorially for the two phases. Their resulting phasor makes an angle of $90^{\circ}$ with the corresponding one for phase A (Figs. 5a and 5b).

The short circuit of phases $\mathrm{B}$ and $\mathrm{C}$ in series can be considered as a single phase in the direction of the resultant phasor (Fig. 5b). If the short circuit occurs in the moment in which the maximum excitation flux links the shorted armature windings, we have a so-called "second characteristic position"[8]. The excitation (secondary) flux $\phi_{2}$ can be divided into useful flux $\left(\phi_{2}\right)$ and leakage flux $\phi_{2 \sigma}$. Assuming for simplicity that the primary and the secondary number of turns are equal i.e., the respective primary and secondary useful inductances are equal, $\mathrm{L}_{\mathrm{u} 1}=\mathrm{L}_{\mathrm{u} 2}=\mathrm{L}_{\mathrm{u}}$, then:

$$
\phi_{2}=\phi_{2 \mathrm{u}}+\phi_{2 \sigma}=\left(\mathrm{L}_{\mathrm{u}}+\mathrm{L}_{2 \sigma}\right) \mathrm{i}_{\mathrm{ex}}=\mathrm{L}_{\mathrm{u}}\left(1+\sigma_{2}\right) \mathrm{i}_{\mathrm{ex}}
$$

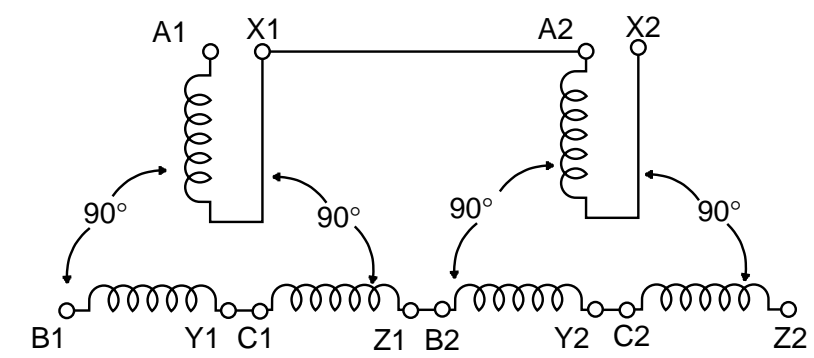

3910.0720

Fig. 4. Disposition of the phase windings (electric angle).

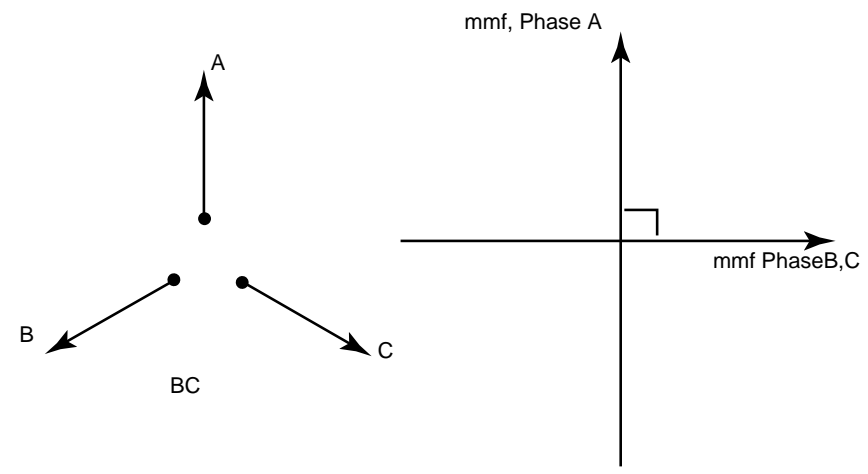

Figs. 5a and 5b. Phasorial relationships between phase windings.
After the moment of short-circuit, the rotor has turned with an angle a, after a time interval of $t=\alpha / \omega$ and the equations of the flux linking the primary and secondary windings are:

$$
\begin{aligned}
& \mathrm{L}_{1 \sigma} \mathrm{i}_{1}+\mathrm{L}_{\chi}\left(\mathrm{i}_{1}+\mathrm{i}_{2} \cos \alpha\right)=\mathrm{L}_{\chi} \mathrm{i}_{\mathrm{ex}} \\
& \mathrm{L}_{2 \sigma} \mathrm{i}_{2}+\mathrm{L}_{\chi}\left(\mathrm{i}_{2}+\mathrm{i}_{1} \cos \alpha\right)=\mathrm{L}_{\chi}\left(1+\sigma_{2}\right) \mathrm{i}_{\mathrm{ex}}
\end{aligned}
$$

where

$$
\begin{aligned}
\mathrm{i}_{1}, \mathrm{i}_{2}, \mathrm{i}_{\mathrm{ex}}= & \text { the instantaneous values for currents in } \\
& \text { the stators and rotor, respectively, and } \\
& \mathrm{i}_{\mathrm{ex}} \text { is the initial rotor current }
\end{aligned}
$$

$\mathrm{L}_{1 \sigma}, \mathrm{L}_{2 \sigma}$, and $\mathrm{L} \chi=$ the leakage inductances for primary, secondary, and, respectively, for the common flux;

$\sigma_{1}, \sigma=$ leakage coefficients.

Using relation (3) we arrive at much simpler equations:

$$
\begin{gathered}
\left(1+\sigma_{1}\right) \mathrm{i}_{1}+\mathrm{i}_{2} \cos \mathrm{a}=\mathrm{i}_{\mathrm{ex}} \\
\mathrm{i}_{1} \cos \alpha+\left(1+\sigma_{2}\right) \mathrm{i}_{2}=\left(1+\sigma_{2}\right) \mathrm{i}_{\mathrm{ex}}
\end{gathered}
$$

Solving these we get :

$$
\begin{aligned}
& \mathrm{i}_{1}=\frac{\left(1+\sigma_{2}\right)(1-\cos \alpha)}{\left(1+\sigma_{1}\right)\left(1+\sigma_{2}\right)-\cos ^{2} \alpha} \mathrm{i}_{\mathrm{ex}} \\
& \mathrm{i}_{2}=\frac{\left(1+\sigma_{1}\right)\left(1+\sigma_{2}\right)-\cos \alpha}{\left(1+\sigma_{1}\right)\left(1+\sigma_{2}\right)-\cos ^{2} \alpha} \mathrm{i}_{\mathrm{ex}}
\end{aligned}
$$

The values become critical for $\cos \alpha=-1,180^{\circ}$ after the short circuit. These values are:

$$
\begin{aligned}
& \mathrm{i}_{1}=\frac{2\left(1+\sigma_{2}\right)}{\left(1+\sigma_{1}\right)\left(1+\sigma_{2}\right)-1} \approx \frac{2\left(1+\sigma_{2}\right)}{\sigma_{1}+\sigma_{2}} \mathrm{i}_{\mathrm{ex}} \\
& \mathrm{i}_{2}=\frac{\left(1+\sigma_{1}\right)\left(1+\sigma_{2}\right)+1}{\left(1+\sigma_{1}\right)\left(1+\sigma_{2}\right)-1} \approx\left[1+\frac{2}{\sigma_{1}+\sigma_{2}}\right] \mathrm{i}_{\mathrm{ex}}
\end{aligned}
$$

The approximate formulations assume that $\sigma_{1}, \sigma_{2} \ll 1$.

The second stage of the system involves inducing the high voltage in the third phase winding AA'. As shown in Figs. 4 and 5, the two short-circuited phases form a winding whose axis is perpendicular to the axis of the high voltage winding AA', and, consequently, they cannot induce the high voltage, being magnetically independent (decoupled). Only the current in the excitation winding (which is on the rotor, in our case) can induce the high voltage. The rotor excitation current attains its maximum value $180^{\circ}$ in the first characteristic position. The maximum instantaneous emf in phase AA' will be reached in this particular moment and its value is: 


$$
\mathrm{V}_{\mathrm{f}, \max , 1}=\left[1+\frac{2}{\sigma}\right] \sqrt{2} \mathrm{~V}
$$

for the maximum initial flux linkage where:

$$
\begin{aligned}
& \mathrm{Vf}=\begin{array}{l}
\text { voltage, maximum value induced in the } \\
\text { output phase, } \\
\mathrm{V}
\end{array} \\
& \sigma=\frac{\phi_{\sigma}}{\phi_{\sigma}+\phi_{\mathrm{u}}}=\begin{array}{l}
\text { value of the phase voltage of the } \\
\text { machine, and }
\end{array} \\
& \begin{array}{l}
\text { ratio between the leakage flux (slot, } \\
\text { end turns, etc. and the total flux - } \\
\text { leakage plus useful). }
\end{array}
\end{aligned}
$$

\section{CONCLUSIONS}

A brief discussion on the issues and benefits of compensation has been presented. The several benefits of compensation include lower machine inductance, lower overvoltages and lower transients in the field coil. Several different ways in which compensation can be provided to enhance certain effects and suppress others has also been suggested. In general, it can be concluded that the effects of compensation are largely positive from the electrical point of view. However, implementation of the compensation presents several problems which are not trivial to overcome. The compensating winding represents an extra spinning mass which must be banded against centrifugal loads. Besides these mechanical forces, there are forces on the compensating winding that are of an electrical origin. These electrical forces in certain regions (edges of poles) try to peel the winding from the surface of the rotor and come from the interaction of the current with the azimuthal component of the excitation field. The banding must therefore provide additional down force to keep the winding under compression at all times. Thus, the mechanical structure of the rotor becomes more complicated and therefore riskier. The implementation of compensation is ultimately a tradeoff that must be made after consideration of all these factors.

\section{REFERENCES}

[1] S. B. Pratap, M. D. Driga, W. F. Weldon, and M. L. Spann, "Future trends for compulsators driving railguns," IEEE Transactions on Magnetics, vol. Mag-22, no. 6, pp. 1681-1683, Nov. 1986.

[2] M. L. Spann, S. B. Pratap, M. D. Werst, W. A. Walls, and C. W. Fulcher*, "Compulsator research at The University of Texas at Austin - an overview," IEEE Transactions on Magnetics, vol. 25, no. 1, Jan. 1989.

[3] S. B. Pratap, K. T. Hsieh, M. D. Driga, and W. F. Weldon, "Advanced compulsator for railguns," IEEE Transactions on Magnetics, vol. 25, no. 1, Jan. 1989.

[4] S.B. Pratap, "Transient eddy current distribution in the shield of the passively compensated compulsator-air core machines," IEEE Transactions on Magnetics, vol. 27, no.4, 1991, pp. 3710-3720.

[5] Austin H. Bonnet, "Insulation systems for PWM inverter-fed motors," IEEE Industry Application Magazine, vol. 4, no. 1, Jan./Feb. 1998, pp. 14-26.

[6] M.G. Say, Alternating Current Machines, John Wiley and Sons, N.Y. 1978, pp. 403-406.

[7] M. D. Driga, Final Report to the U. S. Army, Fort Belvoir RDE Center under contract DAAK-87-P-3597, Dec. 1988.

[8] Rudolf Richter, Electrische Maschinen, Band II, Verlag Birkhanser, Basel-Stuttgart, 1972, pp. 293-298. 
